\title{
FAMILIES WITHOUT MINIMAL NUMBERINGS
}

\section{K. Sh. Abeshev, ${ }^{1}$ S. A. Badaev,${ }^{2}$ and M. Mustafa ${ }^{3}$}

Keywords: computable numbering, Ershov hierarchy, minimal numbering.

It is proved that for any nonzero computable ordinal and its arbitrary notation a, there exists a $\Sigma_{a}^{-1}$-computable family without minimal computable numberings.

\section{INTRODUCTION}

Families without minimal computable numberings are analogs of Blum's speed-up theorem. Two such families of c.e. sets were constructed by V'yugin [1] and Badaev [2], based on different ideas. In the Rogers semilattice of the family built in [1], every element is a least upper bound for two incomparable elements. The family of c.e. sets without minimal computable numberings built in [2] makes essential use of the following criterion of minimality for a (not necessarily computable) numbering.

THEOREM 1 [2]. A numbering $\nu: \omega \rightarrow S$ is minimal if and only if, for every c.e. set $W$, the equality $\nu(W)=S$ entails the existence of a positive equivalence $\varepsilon$ such that

$$
\forall x \forall y((x, y) \in \varepsilon \rightarrow \nu(x)=\nu(y)) \& \forall x \exists y(y \in W \&(x, y) \in \varepsilon) .
$$

The minimality criterion allows us to construct a computable family of c.e. sets without computable minimal numberings by using simple diagonal considerations: if a computable numbering indexes a family then it is not minimal in the family.

The study of computable numberings of families of sets in the Ershov hierarchy revealed new phenomena compared to computable numberings of families of c.e. sets. In [3], for example, we can find a family consisting of two sets, of which one is included in the other, whose Rogers semilattice is

\footnotetext{
${ }^{1}$ Al-Farabi $\quad$ Kazakh $\quad$ National $\quad$ University, Al-Farabi ave. 71, Alma-Ata, 050038 Kazakhstan; kuanqk@gmail.com. ${ }^{2}$ Al-Farabi Kazakh National University, Al-Farabi ave. 71, Alma-Ata, 050038 Kazakhstan; Serikzhan.Badaev@kaznu.kz. ${ }^{3}$ Al-Farabi Kazakh National University, Al-Farabi ave. 71, Alma-Ata, 050038 Kazakhstan; Manat.Mustafa@kaznu.kz.
} 
one-element. In [4], it was shown that a complete analog of Khutoretskii's classical theorem [5] does not hold in the Ershov hierarchy. A family which consists of two disjoint sets and has no principal computable numbering was constructed in [6]. All these results were obtained for d.c.e. sets, i.e., sets in the class $\Sigma_{2}^{-1}$ of the Ershov hierarchy.

Therefore, it is natural to raise questions as to which properties of computable numberings that hold in the classical sense will also hold for computable numberings in the Ershov hierarchy. In this regard, an interesting problem is whether or not there exist families of sets without minimal computable numberings [7, Question 11]. The main result of the present paper is proving the existence of such families in each class of sets from all levels, whether finite or infinite, of the Ershov hierarchy.

THEOREM 2. For every nonzero computable ordinal and its arbitrary ordinal notation $a$, there exists a $\Sigma_{a}^{-1}$-computable family $\mathcal{A}$ of sets that has no $\Sigma_{a}^{-1}$-computable minimal numbering.

Recall some necessary notation and notions. We use Kleene's $\mathcal{O}$, an ordinal notation system (see [8] for details). For $a \in \mathcal{O},|a|_{\mathcal{O}}$ denotes an ordinal for which $a$ is a notation; Kleene's partial ordering on the set $\mathcal{O}$ is denoted by $<_{O}$. Ordinal notations are used for defining infinite levels of the Ershov difference hierarchy introduced initially in [9-11]. However, we use another representation for sets in the Ershov hierarchy, namely, one given in [12].

Let $a$ be a notation for a nonzero computable ordinal. We say that a set $A$ of numbers belongs to the class $\Sigma_{a}^{-1}$ in the Ershov hierarchy if there exist computable functions $f(z, s)$ and $h(z, s)$ such that for all $z, s$, the following conditions hold:

(1) $A(z)=\lim _{s} f(z, s)$ with $f(z, 0)=0$ (hereinafter, $X(z)$ denotes the characteristic function of a set $X)$;

(2) (a) $h(z, 0)=a \& h(z, s+1) \leq_{\mathcal{O}} h(z, s)$;

(b) $f(z, s+1) \neq f(z, s) \Rightarrow h(z, s+1) \neq h(z, s)$.

We call $h$ a change function for $A$ with respect to $f$. A pair $\langle f, h\rangle$ of functions satisfying conditions (1) and (2) is called a $\Sigma_{a}^{-1}$-approximation of a $\Sigma_{a}^{-1}$-set $A$. A numbering of a nonempty set $\mathcal{A}$ is any surjective map $\alpha: \omega \rightarrow \mathcal{A}$. If $\alpha$ and $\beta$ are numberings of $\mathcal{A}$, then we say that $\alpha$ is reducible to $\beta$ (written $\alpha \leqslant \beta$ ) if there is a computable function $f$ such that $\alpha(n)=\beta(f(n))$ for all $n \in \omega$. Two numberings $\alpha$ and $\beta$ are said to be equivalent (written $\alpha \equiv \beta$ ) if $\alpha \leqslant \beta$ and $\beta \leqslant \alpha$. A numbering $\alpha$ of $\mathcal{A}$ is minimal if, for every numbering $\beta$ of $\mathcal{A}, \beta \leqslant \alpha$ implies $\alpha \equiv \beta$.

Following [13], we say that a numbering $\alpha: \omega \longrightarrow \mathcal{A}$ of a family of $\Sigma_{a}^{-1}$-sets $\mathcal{A}$ is $\Sigma_{a}^{-1}$-computable if

$$
\{\langle n, x\rangle: x \in \alpha(n)\} \in \Sigma_{a}^{-1} .
$$

Hence $\alpha$ is a $\Sigma_{a}^{-1}$-computable numbering of a family $\mathcal{A}$ if there exists a $\Sigma_{a}^{-1}$-approximation of a universal set $\{\langle n, x\rangle: x \in \alpha(n)\}$; i.e., there are computable functions $f(n, x, s)$ and $h(n, x, s)$ such that

$$
\alpha(n)(x)=\lim _{s} f(n, x, s), \quad f(n, x, 0)=0
$$


for all $n$ and $x$, and $h(n, x, s)$ is a change function for the set $\{\langle n, x\rangle: x \in \alpha(n)\}$ of a numbering $\alpha$ with respect to $f$.

If $\alpha$ is a $\Sigma_{a}^{-1}$-approximation of a numbering $\alpha$ then $\alpha^{s}$ denotes a numbering whose universal set is

$$
\{\langle n, x\rangle: f(n, x, s)=1\} .
$$

By $\pi$ we denote a computable numbering of the family of all possible $\Sigma_{a}^{-1}$-computable numberings. In correspondence with this numbering is a pair of approximating functions $f_{\pi}(k, n, x, s)$ and $h_{\pi}(k, n, x, s)$ defining $\Sigma_{a}^{-1}$-computability of the sequence $\left\{\pi_{k}\right\}_{k \in \omega}$ of numberings uniformly in $k$. Denote by $p_{\pi}(k, n, x, s)$ a computable function defined as follows.

Put $p_{\pi}(k, n, x, s)=a$ if $h_{\pi}(k, n, x, s)=a$; otherwise, put $p_{\pi}(k, n, x, s)=h_{\pi}(k, n, x, t)$, where $t<s$ is the greatest number for which $h_{\pi}(k, n, x, s)<_{\mathcal{O}} h_{\pi}(k, n, x, t)$. Obviously, $h_{\pi}(k, n, x, s)<_{\mathcal{O}}$ $p_{\pi}(k, n, x, s)$ if $h_{\pi}(k, n, x, s) \neq a$. Furthermore, if $h_{\pi}(k, n, x, s) \neq a$ and $h_{\pi}(k, n, x, s+1) \neq$ $h_{\pi}(k, n, x, s)$ then $p_{\pi}(k, n, x, s+1)<_{\mathcal{O}} p_{\pi}(k, n, x, s)$.

Denote by $\varepsilon_{0}, \varepsilon_{1}, \varepsilon_{2}, \ldots$ a computable numbering of all positive equivalences on $\omega$, and by $\left\{\varepsilon_{m}^{s}\right\}$ a double strongly computable sequence of equivalences on initial segments of the natural series such that for all $m$ and $s$,

$$
\varepsilon_{m}^{s} \subseteq \varepsilon_{m}^{s+1}, \varepsilon_{m}=\bigcup_{s \in \omega} \varepsilon_{m}^{s}
$$

For all undefined notions, we refer the reader to [14].

\section{PROOF OF THE MAIN THEOREM}

We will construct $\mathcal{A}$ as a family indexed by some $\Sigma_{a}^{-1}$-computable numbering $\alpha$, in which each set of $\mathcal{A}$ will have either one or two indices.

Requirements. We build a numbering $\alpha$ and a sequence of c.e. sets $\Pi_{k}, k \in \omega$, meeting the following requirements:

e: $\alpha$ is $\Sigma_{a}^{-1}$-computable;

$\mathcal{P}_{k}:$ if $\pi_{k}$ is a numbering of $\mathcal{A}$ then $\pi_{k}\left(\Pi_{k}\right)=\mathcal{A}$;

$\mathcal{N}_{k, m}:$ if $\pi_{k}$ is a numbering of $\mathcal{A}$ then

$$
\exists x \exists y\left((x, y) \in \varepsilon_{m} \& \pi_{k}(x) \neq \pi_{k}(y)\right) \vee \exists x \forall y \in \Pi_{k}\left((x, y) \notin \varepsilon_{m}\right) .
$$

Now we describe strategies to meet these requirements.

Strategy for $\mathcal{C}$. We construct a pair of computable numberings $f(n, x, s)$ and $g(n, x, s)$ in stages to form a $\Sigma_{a}^{-1}$-approximation to a numbering $\alpha$. In fact, we could well do without even mentioning $\alpha$ since $\alpha$ is defined uniquely by the pair of functions $f, g$. However, it seems more convenient to give an informal explanation of the ideas behind our construction in terms of the numbering $\alpha$. 
In the numbering $\alpha$, each pair of consecutive indices $2 x, 2 x+1$ is intended to meet exactly one requirement $\mathcal{N}_{k, m}$. For this reason, we will consider the number $x$ as the standard index $\langle k, m\rangle$ of some pair $(k, m)$. Sets $\alpha(2 x)$ and $\alpha(2 x+1)$ will have some common static part, but each of these will also have its own dynamically changeable part. At each stage of the construction below, the dynamic part of a set consists exactly of two numbers. At each stage, one element of the dynamic part of each of the sets $\alpha(2 x)$ and $\alpha(2 x+1)$ can be moved into the common static part of these sets, but no number of the static part of a set can be moved into its dynamic part. In addition, two numbers of sets $\alpha(2 y)$ and $\alpha(2 y+1)$ with $y \neq x$ can be enumerated simultaneously into both sets $\alpha(2 x)$ and $\alpha(2 x+1)$, and then can be extracted simultaneously from both $\alpha(2 x)$ and $\alpha(2 x+1)$. In the limit, two sets $\alpha(2 x)$ and $\alpha(2 x+1)$ may coincide or be distinct, depending on whether the process of moving elements from the dynamic parts into the static part is infinite or finite. At all nonzero stages, the static part of a pair of sets $\alpha(2 x), \alpha(2 x+1)$ is not included into the static part of any $\alpha(z), z \notin\{2 x, 2 x+1\}$, and at stage 1 , the static parts of $\alpha(2 x)$ and $\alpha(z)$ are nonempty and disjoint. Thus, in the numbering $\alpha$, each set of $\mathcal{A}$ will have either one or two indices; in the latter case, these two indices are consecutive natural numbers of the form $2 x, 2 x+1$. We describe in precise terms the process showing how both sets $\alpha(2 x)$ and $\alpha(2 x+1)$ increase with increasing their dynamic parts.

Fix three arbitrary injective computable functions $d_{0}: \omega^{2} \mapsto \omega, d_{1}: \omega^{2} \mapsto \omega$, and $\sigma: \omega^{3} \mapsto \omega$ with pairwise disjoint ranges. For every $x$, values for the function $\lambda e \lambda s \sigma(x, e, s)$ form the static part of the sets $\alpha(2 x)$ and $\alpha(2 x+1)$, while some (or all) values of the function $\lambda s d_{j}(x, s)$ are enumerated step by step into the dynamic part of $\alpha(2 x+j)$, where $j \leq 1$. At the beginning of the construction, for each $x$, we put

$$
\begin{aligned}
& \alpha^{0}(2 x)=\alpha^{0}(2 x+1)=\varnothing, \\
& \alpha^{1}(2 x)=\{\sigma(x, e, i): e, i \in \omega\} \cup\left\{d_{0}(x, 0), d_{0}(x, 1)\right\}, \\
& \alpha^{1}(2 x+1)=\{\sigma(x, e, i): e, i \in \omega\} \cup\left\{d_{1}(x, 0), d_{1}(x, 1)\right\} .
\end{aligned}
$$

Then we declare the numbers $d_{0}(x, 0)$ and $d_{1}(x, 0)$ to be active and declare the numbers $d_{0}(x, 1)$ and $d_{1}(x, 1)$ to be semi-active.

The process of changing the dynamic parts of $\alpha(2 x)$ and $\alpha(2 x+1)$ (under certain conditions) begins with the following actions:

the number $d_{0}(x, 0)$ is enumerated into the static part of $\alpha(2 x+1)$ and thereafter ceases to be active;

the number $d_{1}(x, 0)$ is enumerated into the static part of $\alpha(2 x)$ and thereafter ceases to be active;

the semi-active numbers $d_{0}(x, 1)$ and $d_{1}(x, 1)$ are declared active;

the number $d_{0}(x, 2)$ is enumerated into the dynamic part of $\alpha(2 x)$ and is declared semi-active;

the number $d_{1}(x, 2)$ is enumerated into the dynamic part of $\alpha(2 x+1)$ and is declared semiactive.

Thus, as a result of these actions, a pair of active numbers is moved from the dynamic parts 
of the sets $\alpha(2 x)$ and $\alpha(2 x+1)$ into their static part and ceases to be active; a pair of semi-active numbers is activated; the next two unused numbers are declared semi-active, and one of them is enumerated into the dynamic part of $\alpha(2 x)$, and the other into the dynamic part of $\alpha(2 x+1)$.

Changing the dynamic parts of $\alpha(2 x)$ and $\alpha(2 x+1)$ proceeds as an iterative process. An iteration step for a pair of active numbers $d_{0}(x, i)$ and $d_{1}(x, i)$ is described in the following:

Procedure $\mathcal{D}(x, i)$ :

the number $d_{0}(x, i)$ is enumerated into $\alpha(2 x+1)$ and thereafter ceases to be active;

the number $d_{1}(x, i)$ is enumerated into $\alpha(2 x)$ and thereafter ceases to be active;

the semi-active numbers $d_{0}(x, i+1)$ and $d_{1}(x, i+1)$ are declared active;

the number $d_{0}(x, i+2)$ is enumerated into $\alpha(2 x)$ and is declared semi-active;

the number $d_{1}(x, i+2)$ is enumerated into $\alpha(2 x+1)$ and is declared semi-active.

Without loss of generality, we may assume that $\pi_{0}^{s}(y)=\varnothing$ for all $s$ and $y$. By our construction, for every $x$, none of $\sigma(x, 0, i), i \in \omega$, is extracted from $\alpha(2 x)$ and $\alpha(2 x+1)$, nor is enumerated into $\alpha(2 y)$ and $\alpha(2 y+1)$ for $x \neq y$. Therefore,

$$
\{\alpha(2 x), \alpha(2 x+1)\} \cap\{\alpha(2 y), \alpha(2 y+1)\}=\varnothing
$$

for any distinct numbers $x$ and $y$.

Strategy for $\mathcal{P}_{k}$ in isolation. For every $k$, we split the set $\Pi_{k}$ of $\pi_{k}$-indices into three parts: $L_{k}, R_{k}$, and $O_{k}$. If $\pi_{k}$ is a numbering of $\mathcal{A}$, then we require that

$L_{k}$ contain exactly one $\pi_{k}$-index for every set $\alpha(2 x)$, with $\langle x\rangle_{0}=k$;

$R_{k}$ contain exactly one $\pi_{k}$-index for every set $\alpha(2 x+1)$, with $\langle x\rangle_{0}=k$ and $\alpha(2 x) \neq \alpha(2 x+1)$;

$O_{k}$ consist of all $\pi_{k}$-indices of the remaining sets, i.e., all sets of the form $\alpha(2 z)$ or $\alpha(2 z+1)$, with $\langle z\rangle_{0} \neq k$.

Note that in this case the set $\Pi_{k}=L_{k} \cup R_{k} \cup O_{k}$ will contain exactly one $\pi_{k}$-index for each set of the subfamily

$$
\left\{\alpha(2 x), \alpha(2 x+1):\langle x\rangle_{0}=k\right\} .
$$

Our idea is that the equality $\alpha(2 x)=\alpha(2 x+1)$ could occur only in the limit. An important point in the strategy for $\mathcal{P}_{k}$ is the following: if $\alpha(2 x) \neq \alpha(2 x+1)$ then this inequality is explicitly recognized at a finite stage.

Clearly, the above requirements for the strategy for $\mathcal{P}_{k}$ ensure that $\alpha\left(\Pi_{k}\right)=\mathcal{A}$. Also, for the set $\Pi_{k}$ to be c.e., it is necessary that the requirements have a computable realization. The sets $L_{k}$, $R_{k}$, and $O_{k}$ will consist of some values for partial computable functions $l(x), r(x)$, and $o(x, e, i)$ defined as follows. Let $x=\langle k, m\rangle_{0}$. In the approximation $\pi_{k}^{s}$, we choose two distinct $\pi_{k}$-indices $y_{0}$ and $y_{1}$ such that $d_{0}(x, 0) \in \pi_{k}^{s}\left(y_{0}\right)$ and $d_{1}(x, 0) \in \pi_{k}^{s}\left(y_{1}\right)$; put $l(x)=y_{0}$ and $r(x)=y_{1}$. For every pair $(e, i)$ of numbers, where $e \neq k$, we define $o(x, e, i)=i$ if $\sigma(x, e, i) \in \pi_{e}(i)$. Note that

$$
o(x, e, i) \downarrow \Rightarrow \sigma(x, e, i) \in \pi_{e}(i) \& o(x, e, i)=i .
$$


Put

$$
\begin{aligned}
L_{k} & =\left\{l(x):\langle x\rangle_{0}=k\right\}, \\
R_{k} & =\left\{r(x):\langle x\rangle_{0}=k \& \alpha(2 x) \neq \alpha(2 x+1)\right\}, \\
O_{k} & =\left\{o(x, k, i):(x, k, i) \in \operatorname{dom}(o) \&\langle x\rangle_{0} \neq k\right\} .
\end{aligned}
$$

When the values $l(x), r(x)$, and $o(x, e, i)$ are defined, we should take care that these values be correct. For a numbering $\pi_{k}$ of the family $\mathcal{A}$, below we show how to ensure that $\pi_{k}(o(x, k, i)) \in$ $\{\alpha(2 x), \alpha(2 x+1)\}$ if $\langle x\rangle_{0} \neq k$, and that $\alpha(2 x)=\pi_{k}(l(x))$ and $\alpha(2 x+1)=\pi_{k}(r(x))$ if $\langle x\rangle_{0}=k$.

Procedure $\mathcal{O}(x, e, i)$. This procedure can be performed at some stage $s$ of the construction only if the value $o(x, e, i)$ has been defined by stage $s$. Thus, if the inactive number $\sigma(x, e, i)$ is not in the set $\pi_{e}(i)$ at stage $s$, then we proceed as follows:

(1) enumerate $\sigma(x, e, i)$ into all sets $\alpha(z), z \notin\{2 x, 2 x+1\}$;

(2) wait for $\sigma(x, e, i)$ to appear in $\pi_{e}(i)$ at some stage $s^{\prime}>s$;

(3) at stage $s^{\prime}$, remove the number $\sigma(x, e, i)$ from all sets $\alpha(z), z \notin\{2 x, 2 x+1\}$, and thereby complete the procedure.

If $\pi_{k}$ is a numbering of $\mathcal{A}$ and $o(x, k, i)$ is defined then the procedure $\mathcal{O}(x, k, i)$ guarantees that $\pi_{k}(i)$ will be one of the sets $\alpha(2 x)$ or $\alpha(2 x+1)$. Indeed, at stage 0 , the number $\sigma(x, k, i)$ is enumerated into both sets $\alpha(2 x)$ and $\alpha(2 x+1)$, never to leave them. Until the moment when the value $o(x, k, i)$ is defined, the number $\sigma(x, k, i)$ cannot enter the sets $\alpha(2 z)$ and $\alpha(2 z+1)$ for all $z \neq x$. At the moment when the value $o(x, k, i)$ becomes defined in the construction, the number $\sigma(x, k, i)$ is in the set $\pi_{k}(i)$. If $\sigma(x, k, i)$ is not removed from $\pi_{k}(i)$ at later stages, then $\pi_{k}(i) \in\{\alpha(2 x), \alpha(2 x+1)\}$, since $\sigma(x, k, i)$ is not contained in the sets $\alpha(2 z)$ and $\alpha(2 z+1)$ for $z \neq x$. On the other hand, if the number $\sigma(x, k, i)$ leaves $\pi_{k}(i)$ after stage $s$, then either it will come back to $\pi_{k}(i)$ later or the procedure $\mathcal{O}(x, e, i)$ will be performed. After item (1) of the procedure is effected, all sets of the family $\mathcal{A}$ will contain the number $\sigma(x, k, i)$; since $\pi_{k}(i) \in \mathcal{A}$, 'waiting' in item (2) will be successful, and by item (3), the number $\sigma(x, k, i)$ will be removed from all sets $\alpha(2 z)$ and $\alpha(2 z+1)$ for $z \neq x$. Clearly, the procedure $\mathcal{O}(x, e, i)$ cannot be performed infinitely many times. Hence $\pi_{k}(i) \in\{\alpha(2 x), \alpha(2 x+1)\}$.

In fact, we have shown that if $\pi_{k}$ is a numbering of $\mathcal{A}$ then $o(x, k, i)$ is defined and

$$
\sigma(x, k, i) \in \pi_{k}(i) \Longleftrightarrow \pi_{k}(i) \in\{\alpha(2 x), \alpha(2 x+1)\} .
$$

Procedure $\mathcal{L}(x, i)$. This procedure is performed for the active number $d_{0}(x, i)$ and the set $\pi_{k}(l(x))$, where $k=\langle x\rangle_{0}$. Recall that the number $d_{0}(x, i)$ active at stage $s$ is contained in $\alpha^{s}(2 x)$ and is not contained in $\alpha^{s}(2 x+1)$.

Thus if the active number $d_{0}(x, i)$ is not in $\pi_{k}(l(x))$ at stage $s$, then we proceed as follows:

(1) enumerate $d_{0}(x, i)$ into all sets $\alpha(z), z \neq 2 x$;

(2) wait until $d_{0}(x, i)$ is enumerated into $\pi_{k}(l(x))$ at some stage $s^{\prime}>s$; 
(3) at stage $s^{\prime}$, remove the number $d_{0}(x, i)$ from all sets $\alpha(z), z \neq 2 x$.

It is not difficult to show that if the number $d_{0}(x, i)$ is declared active at some stage and remains active afterwards, then $\alpha(2 x)=\pi_{k}(l(x))$.

Procedure $\mathcal{R}(x, i)$. This procedure derives from the procedure $\mathcal{L}(x, i)$, with the number $d_{0}(x, i)$ and the set $\pi_{k}(l(x))$ replaced by $d_{1}(x, i)$ and $\pi_{k}(r(x))$, respectively.

A priori, we may need to simultaneously perform two or more procedures such as above, which is apt to lead to conflicts. To avoid these, we can easily order (in a suitable priority list) the procedures performed with elements of $\alpha(2 x)$ and $\alpha(2 x+1)$ so that, at every stage, no more than one procedure is carried out, and item (1) of every next procedure starts only after the completion of item (3) in the previous procedure. Note that in the case where $\pi_{k}$ is a numbering of $\mathcal{A}$, if a procedure starts then it will eventually be completed.

Thus our strategy to meet the requirement $\mathcal{P}_{k}$ is the following:

do not extract inactive numbers from those sets of $\mathcal{A}$ into which these numbers are enumerated for the first time;

for every $i \leq 1$, do not extract active numbers $d_{i}(x, j), j \in \omega$, from the set $\alpha(2 x+i)$;

perform the procedure $\mathcal{O}(x, e, i)$ whenever it is necessary and does not lead to conflict;

perform the procedure $\mathcal{L}(x, i)$ whenever it is necessary and does not lead to conflict;

perform the procedure $\mathcal{R}(x, i)$ whenever it is necessary and does not lead to conflict.

It only remains to describe a mechanism for recognizing the moment when, in constructing the set $R_{k}$, we could ensure that $\alpha(2 x) \neq \alpha(2 x+1)$. We can do this right now if we note that

$$
R_{k}=\left\{r(x):\langle x\rangle_{0}=k \&(l(x), r(x)) \in \varepsilon_{\langle x\rangle_{1}}\right\} .
$$

The reason why this equality holds will be explained in describing the following strategy.

Strategy for $\mathcal{N}_{k, m}$ in isolation. To meet the requirement $\mathcal{N}_{k, m}$, we use two $\pi_{k}$-indices $l(x)$ and $r(x)$ of the sets $\alpha(2 x)$ and $\alpha(2 x+1)$, where $x=\langle k, m\rangle$. Under the assumption that $\pi_{k}$ is a numbering of $\mathcal{A}$, we wait for the values $l(x)$ and $r(x)$ to be defined as in the strategy for $\mathcal{P}_{k}$ above, and then begin to enumerate ever more new numbers into the sets $\alpha(2 x)$ and $\alpha(2 x+1)$ through their dynamic parts, so that $\alpha(2 x)$ and $\alpha(2 x+1)$ could become equal in the limit (remaining distinct at each finite stage) and simultaneously check whether the pair $(l(x), r(x))$ appears in the enumeration of $\varepsilon(m)$. As soon as it does, we stop changing $\alpha(2 x)$ and $\alpha(2 x+1)$. It remains to ensure that in the limit,

$$
\alpha(2 x)=\pi_{k}(l(x)), \quad \alpha(2 x+1)=\pi_{k}(r(x)) .
$$

For every $x=\langle k, m\rangle$ fixed, we execute the procedure $\mathcal{N}(k, m)$ to meet the requirement $\mathcal{N}_{k, m}$ : namely, at each step $s$, for the active numbers $d_{0}(x, i)$ and $d_{1}(x, i)$,

check whether the pair $(l(x), r(x))$ of numbers belongs to the set $\varepsilon^{s}(m)$;

check whether the following two inclusions hold:

$$
\left\{d_{0}(x, i), d_{0}(x, i+1)\right\} \subseteq \pi_{\langle x\rangle_{0}}^{s}(l(x)),
$$




$$
\left\{d_{1}(x, i), d_{1}(x, i+1)\right\} \subseteq \pi_{\langle x\rangle_{0}}^{s}(r(x))
$$

the procedure $\mathcal{D}(x, i)$ is executed iff the answer to the first question is negative, while the answer to the second one is affirmative.

Conflicts between strategies. Obviously, strategies of the same type cannot conflict with each other, since their instructions are executed for distinct pairs of sets. For the same reason, there are no conflicts between strategies $\mathcal{P}_{k^{\prime}}$ and $\mathcal{N}_{k, m}$ if $k^{\prime} \neq k$.

Formally, a conflict between strategies $\mathcal{P}_{k}$ and $\mathcal{N}_{k, m}$ arises in the following situation: there is a same stage at which the conditions for applying the procedures $\mathcal{L}(x, i)$ or $\mathcal{R}(x, i)$ on the one hand and the procedure $\mathcal{N}(k, m)$ on the other hand hold for the same pair of sets $\alpha(2 x), \alpha(2 x+1)$, where $x=\langle k, m\rangle$. In such situations, we have the following priority list for the execution of the procedures (from higher to lower): $\mathcal{L}(x, i), \mathcal{R}(x, i), \mathcal{D}(x, i)$.

Construction. We build in stages $s$ computable functions $f(x, y, s)$ and $h(x, y, s)$ and partial computable functions $o(x, e, i), l(x)$, and $r(x)$.

Stage $s=0$. Let $f(x, y, 0)=0$ and $h(x, y, 0)=a$ for all $x$ and $y$. The values $o(x, e, i), l(x)$, and $r(x)$ are undefined for all $x, e, i$.

Below we assume by default that $f(x, y, s+1)=f(x, y, s)$ and $h(x, y, s+1)=h(x, y, s)$ for all those $x, y$ for which the values of $f(x, y, s+1)$ and $h(x, y, s+1)$ are not explicitly defined at stage $s+1$.

Stage $s=1$. For each $x$ and for all $y \in\{\sigma(x, e, i): e, i \in \omega\} \cup\left\{d_{0}(x, 0), d_{0}(x, 1)\right\}$, put $f(2 x, y, 1)=1$ and $h(2 x, y, 1)=1$. For each $x$ and for all $y \in\{\sigma(x, e, i): e, i \in$ $\omega\} \cup\left\{d_{1}(x, 0), d_{1}(x, 1)\right\}$, let $f(2 x+1, y, 1)=1$ and $h(2 x+1, y, 1)=1$. The numbers $d_{0}(x, 0)$ and $d_{1}(x, 0)$ are declared active, while $d_{0}(x, 1)$ and $d_{1}(x, 1)$ are declared semi-active.

Stage $s+1, s>0$. Let $\langle s\rangle_{0}=\langle x, e, i\rangle, x=\langle k, m\rangle$. We follow the instructions of 10 steps below, one by one, unless there is an explicit command to go to another step. If the conditions of a step do not hold, then by default we pass to the next step.

(1) If $k \neq e, o(x, e, i)$ is undefined, and $f_{\pi}(e, i, \sigma(x, e, i), s+1)=1$, then put $o(x, e, i)=i$.

(2) If $k \neq e, o(x, e, i)$ is defined, $f_{\pi}(e, i, \sigma(x, e, i), s+1)=0$, and $f(2 x+2, \sigma(x, e, i), s)=0$, then let

$$
f(z, \sigma(x, e, i), s+1)=1, h(z, \sigma(x, e, i), s+1)=h_{\pi}(e, i, \sigma(x, e, i), s+1)
$$

for all $z \notin\{2 x, 2 x+1\}$.

(3) If $k \neq e, o(x, e, i)$ is defined, $f_{\pi}(e, i, \sigma(x, e, i), s+1)=1$, and $f(2 x+2, \sigma(x, e, i), s)=1$, then put

$$
f(z, \sigma(x, e, i), s+1)=0, h(z, \sigma(x, e, i), s+1)=h_{\pi}(e, i, \sigma(x, e, i), s+1)
$$

for all $z \notin\{2 x, 2 x+1\}$.

(4) If $k=e, l(x)$ and $r(x)$ are undefined, $f_{\pi}\left(k, y_{0}, d_{0}(x, 0), s+1\right)=1$, and $f_{\pi}\left(k, y_{1}, d_{1}(x, 0), s+\right.$ $1)=1$ for some distinct $y_{0}$ and $y_{1}$, then choose the least such $y_{0}$ and $y_{1}$ and define $l(x)=y_{0}$ and $r(x)=y_{1}$. 
(5) If $k=e, l(x)$ and $r(x)$ are defined, the number $d_{0}(x, i)$ is active or has been active, and

$$
f_{\pi}\left(k, l(x), d_{0}(x, i), s+1\right)=f\left(2 x+2, d_{0}(x, i), s\right)=0,
$$

then let

$$
f\left(z, d_{0}(x, i), s+1\right)=1, h\left(z, d_{0}(x, i), s+1\right)=p_{\pi}\left(k, l(x), d_{0}(x, i), s+1\right)
$$

for all $z \neq 2 x$, if $d_{0}(x, i)$ is active, and for all $z \notin\{2 x, 2 x+1\}$ if $d_{0}(x, i)$ is not active. Go to step (10).

(6) If $k=e, l(x)$ and $r(x)$ are defined, the number $d_{0}(x, i)$ is active or has been active, and

$$
f_{\pi}\left(k, l(x), d_{0}(x, i), s+1\right)=f\left(2 x+2, d_{0}(x, i), s\right)=1,
$$

then put

$$
f\left(z, d_{0}(x, i), s+1\right)=0, h\left(z, d_{0}(x, i), s+1\right)=p_{\pi}\left(k, l(x), d_{0}(x, i), s+1\right)
$$

for all $z \neq 2 x$, if $d_{0}(x, i)$ is active, and for all $z \notin\{2 x, 2 x+1\}$ if $d_{0}(x, i)$ is not active, and go to step (7). Otherwise, go to step (10).

(7) If $k=e, l(x)$ and $r(x)$ are defined, the number $d_{1}(x, i)$ is active or has been active, and

$$
f_{\pi}\left(k, r(x), d_{1}(x, i), s+1\right)=f\left(2 x+2, d_{1}(x, i), s\right)=0,
$$

then let

$$
f\left(z, d_{1}(x, i), s+1\right)=1, h\left(z, d_{1}(x, i), s+1\right)=p_{\pi}\left(k, r(x), d_{1}(x, i), s+1\right)
$$

for all $z \neq 2 x+1$, if $d_{1}(x, i)$ is active, and for all $z \notin\{2 x, 2 x+1\}$ if $d_{1}(x, i)$ is not active. Go to step (10).

(8) If $k=e, l(x)$ and $r(x)$ are defined, the number $d_{1}(x, i)$ is active or has been active, and

$$
f_{\pi}\left(k, r(x), d_{1}(x, i), s+1\right)=f\left(2 x+2, d_{1}(x, i), s\right)=1,
$$

then let

$$
f\left(z, d_{1}(x, i), s+1\right)=0, h\left(z, d_{1}(x, i), s+1\right)=p_{\pi}\left(k, r(x), d_{1}(x, i), s+1\right)
$$

for all $z \neq 2 x+1$, if $d_{1}(x, i)$ is active, and for all $z \notin\{2 x, 2 x+1\}$ if $d_{1}(x, i)$ is not active, and go to step (9). Otherwise, go to step (10).

(9) If $k=e, l(x)$ and $r(x)$ are defined, $(l(x), r(x)) \notin \varepsilon_{m}^{s+1}$, the numbers $d_{0}(x, i)$ and $d_{1}(x, i)$ are active, and

$$
\begin{aligned}
f_{\pi}\left(k, l(x), d_{0}(x, i), s+1\right) & =f_{\pi}\left(k, l(x), d_{0}(x, i+1), s+1\right)=1, \\
f_{\pi}\left(k, r(x), d_{1}(x, i), s+1\right) & =f_{\pi}\left(k, r(x), d_{1}(x, i+1), s+1\right)=1, \\
f\left(2 x, d_{1}(x, i), s\right) & =f\left(2 x+1, d_{0}(x, i), s\right)=0
\end{aligned}
$$


then put

$$
\begin{gathered}
f\left(2 x, d_{1}(x, i), s+1\right)=1, f\left(2 x+1, d_{0}(x, i), s+1\right)=1, \\
h\left(2 x, d_{1}(x, i), s+1\right)=1, h\left(2 x+1, d_{0}(x, i), s+1\right)=1
\end{gathered}
$$

and declare the numbers $d_{0}(x, i)$ and $d_{1}(x, i)$ to be inactive. Declare the semi-active numbers $d_{0}(x, i+1)$ and $d_{1}(x, i+1)$ to be active. Define

$$
\begin{aligned}
& f\left(2 x, d_{0}(x, i+2), s+1\right)=1, f\left(2 x+1, d_{1}(x, i+2), s+1\right)=1, \\
& h\left(2 x, d_{0}(x, i+2), s+1\right)=1, h\left(2 x+1, d_{1}(x, i+2), s+1\right)=1
\end{aligned}
$$

and declare the numbers $d_{0}(x, i+2)$ and $d_{1}(x, i+2)$ to be semi-active.

(10) Go to the next stage.

The construction is completely described. Clearly, steps (2) and (3) of the construction correspond to the procedure $\mathcal{O}(x, e, i)$, steps $(5)$ and $(6)$ to the procedure $\mathcal{L}(x, i)$, steps $(7)$ and $(8)$ to the procedure $\mathcal{R}(x, i)$, and step (9) aims to meet the requirement $\mathcal{N}_{k, m}$.

Obviously, the functions $f$ and $h$ are computable, while $l, r$, and $o$ are partial computable. Define a numbering $\alpha$ by setting $\alpha(n)(y)=\lim _{s} f(n, y, s)$ for all $n$ and $y$. Let $\mathcal{A}=\{\alpha(n): n \in \omega\}$. Denote by $S(x, e, i)$ the set of all stages $s+1^{s}$ for which $\langle s\rangle_{0}=\langle x, e, i\rangle$.

The proof of the theorem proceeds via several lemmas.

LEMMA 1. A pair $\langle f, h\rangle$ of functions is a $\Sigma_{a}^{-1}$-approximation to a numbering $\alpha$, and hence $\alpha$ is $\Sigma_{a}^{-1}$-computable.

Proof. It is sufficient to verify that $h$ is a change function for $\alpha$. By construction, $h(n, y, 0)=a$ for all $n$ and $y$. We are left to verify that for each pair of numbers $n, y$ and for all $s$, the following two properties hold:

(i) $h(n, y, s+1) \leq_{\mathcal{O}} h(n, y, s)$;

(ii) $f(n, y, s+1) \neq f(n, y, s) \Rightarrow h(n, y, s+1) \neq h(n, y, s)$.

Properties (i) and (ii) are evident for every $s$ and for all pairs $(n, y)$ like

$n=2 x, y \in\{\sigma(x, e, i): e, i \in \omega\} \cup\left\{d_{0}(x, i): i \in \omega\right\}$;

$n=2 x+1, y \in\{\sigma(x, e, i): e, i \in \omega\} \cup\left\{d_{1}(x, i): i \in \omega\right\}$;

$n \in \omega, y \notin \operatorname{range}\left(d_{0}\right) \cup \operatorname{range}\left(d_{1}\right) \cup \operatorname{range}(\sigma)$.

We verify these properties for the other pairs.

For every $x$, if $y \in\{\sigma(x, e, i): e, i \in \omega\}$ and $n \notin\{2 x, 2 x+1\}$, then the function $\lambda s h(n, y, s)$ can change its values only at stages $s+1 \in S(x, e, i)$, for which $x=\langle k, m\rangle$ and $k \neq e$, as a result of executing steps (2) and (3) of the construction. At such steps, $h(n, y, s+1)=h_{\pi}(e, i, y, s)$, and so properties (i) and (ii) for the function $\lambda s h(n, y, s)$ follow from those for $\lambda s h_{\pi}(e, i, y, s)$ in virtue of $\leq_{\mathcal{O}}$ being a transitive relation.

Now we consider the case where $(n, y)$ is a pair with $n \neq 2 x$ and $y=d_{0}(x, i)$ for arbitrary $x$ and $i$. If the number $d_{0}(x, i)$ never becomes active or $x \notin \operatorname{dom}(l)$, then $f(n, y, s)=0$ and $h(n, y, s)=a$ for all $s$, and hence there is nothing to prove. 
Let $x=\langle k, m\rangle$ for some $m$ and suppose that at stage $s_{0}+1 \in S(x, k, i)$ the active number $d_{0}(x, i)$ is enumerated for the first time into $\alpha(n)$ for every $n \neq 2 x$ due to step (5). Then $f\left(n, d_{0}(x, i), s_{o}\right)=$ $0, h\left(n, d_{0}(x, i), s_{o}\right)=a$ and $f\left(n, d_{0}(x, i), s_{o}+1\right)=1, h\left(n, d_{0}(x, i), s_{o}+1\right)=p_{\pi}\left(k, l(x), d_{0}(x, i), s_{0}+\right.$ $1)$. We show that $h\left(n, d_{0}(x, i), s_{o}+1\right)<_{\mathcal{O}} h\left(n, d_{0}(x, i), s_{o}\right)$. Indeed, at stage $s_{0}+1$, the value $l(x)$ is already defined. Let $s_{1}+1$ be a stage at which this value became defined. Then $s_{1}<s_{0}$, and at stage $s_{1}+1$, step (4) holds and $f_{\pi}\left(k, l(x), d_{0}(x, 0), s_{1}+1\right)=1$. Therefore, by stage $s_{0}+1$, the function $\lambda s p_{\pi}\left(k, l(x), d_{0}(x, i), s\right)$ has changed its value at least twice. This implies that

$$
p_{\pi}\left(k, l(x), d_{0}(x, i), s_{0}+1\right)<_{\mathcal{O}} p_{\pi}\left(k, l(x), d_{0}(x, i), s_{1}+1\right) \leq_{\mathcal{O}} a .
$$

Hence $h\left(n, d_{0}(x, i), s_{o}+1\right)<_{\mathcal{O}} h\left(n, d_{0}(x, i), s_{o}\right)$.

Thus properties (i) and (ii) hold for the desired pairs if $s \leq s_{0}$. For all pairs $\left\langle n, d_{0}(x, i)\right\rangle$, if $n \notin\{2 x, 2 x+1\}$ and $s \leq s_{0}$, then properties (i) and (ii) for the function $\lambda \operatorname{sh}\left(n, d_{0}(x, i), s\right)$ follow from those for $\lambda s p_{\pi}\left(k, l(x), d_{0}(x, i), s\right)$.

After stage $s_{0}$ up to stage $s_{2}+1$ at which the number $d_{0}(x, i)$ is enumerated into the set $\alpha(2 x+1)$ via the procedure $\mathcal{D}(x, i)$ (if ever), satisfaction of (i) and (ii) for the function $\lambda \operatorname{sh}\left(2 x+1, d_{0}(x, i), s\right)$ follows from the properties of the function $\lambda s p_{\pi}\left(k, l(x), d_{0}(x, i), s\right)$.

Thus we need to consider properties (i) and (ii) for $s=s_{2}$. According to the instructions of step (9), $f\left(2 x+1, d_{0}(x, i), s_{2}\right)=0, f\left(2 x+1, d_{0}(x, i), s_{2}+1\right)=1$, and $h\left(2 x+1, d_{0}(x, i), s_{2}+1\right)=1$. Furthermore,

$$
p_{\pi}\left(k, l(x), d_{0}(x, 0), s_{2}\right) \leq_{\mathcal{O}} h\left(2 x+1, d_{0}(x, i), s_{2}\right),
$$

if, by stage $s_{2}+1, d_{0}(x, i)$ has been enumerated at least once into $\alpha(2 x+1)$ due to step (5) of the construction, and $h\left(2 x+1, d_{0}(x, i), s_{2}\right)=a$ otherwise. This, together with the inequalities

$$
1 \leq_{\mathcal{O}} h_{\pi}\left(k, l(x), d_{0}(x, 0), s_{2}\right)<_{\mathcal{O}} p_{\pi}\left(k, l(x), d_{0}(x, 0), s_{2}\right),
$$

yields $h\left(2 x+1, d_{0}(x, i), s_{2}+1\right)<_{\mathcal{O}} h\left(2 x+1, d_{0}(x, i), s_{2}\right)$. Hence properties (i) and (ii) hold for $s=s_{2}$ as well. Finally, $f\left(2 x+1, d_{0}(x, i), s\right)=h\left(2 x+1, d_{0}(x, i), s\right)=1$ for all $s \geq s_{2}+1$. Thus properties (i) and (ii) are also true for the pair $\left\langle 2 x+1, d_{0}(x, i)\right\rangle$ with all $s$.

In the symmetric case where $n \neq 2 x+1$ and $y=d_{1}(x, i)$, properties (i) and (ii) can be proved in a similar way. Lemma 1 is completed.

LEMMA 2. If $\pi_{k}$ is a numbering of the family $\mathcal{A}$, then, for any $x$ and $i$ with $\langle x\rangle_{0} \neq k$, the value $o(x, k, i)$ is defined if and only if $\pi_{k}(i)=\alpha(2 x)$ or $\pi_{k}(i)=\alpha(2 x+1)$.

Proof. Necessity. Let $x, i$ be any pair of numbers such that $\langle x\rangle_{0} \neq k$ and the value $o(x, k, i)$ is defined. Consider a stage $s_{0}+1 \in S(x, k, i)$ at which the value $o(x, k, i)$ became defined. This is possible only by virtue of the execution of step (1) at this stage. Then $f_{\pi}\left(k, i, \sigma(x, k, i), s_{0}+\right.$ $1)=1$, i.e., $\sigma(x, k, i) \in \pi_{k}^{s_{0}+1}(i)$. By construction, the number $\sigma(x, k, i)$ is enumerated due to the instructions of step 1 into the sets $\alpha(2 x)$ and $\alpha(2 x+1)$ only; later on, it can be enumerated into other sets of $\mathcal{A}$ only via step (2). If step (2) is not performed at all stages of $S(x, k, i)$ then $\pi_{k}(i)=\alpha(2 x)$ or $\pi_{k}(i)=\alpha(2 x+1)$. 
Let $s_{1}+1 \in S(x, k, i)$ be the least stage at which step (2) holds. Then $s_{1}>s_{0}$ and

$$
f_{\pi}\left(k, i, \sigma(x, k, i), s_{1}+1\right)=0 \text { and } f\left(z, \sigma(x, k, i), s_{1}+1\right)=1
$$

for every $z$. This means that at stage $s_{1}+1$ the number $\sigma(x, k, i)$ is contained in all sets of the family $\mathcal{A}$ except the set $\pi_{k}(i)$. Since $\pi_{k}$ is a numbering of $\mathcal{A}$, it follows that at some later stage the number $\sigma(x, k, i)$ will be enumerated into $\pi_{k}(i)$ again. Let $s_{2}+1 \in S(x, k, i)$ be the least stage such that $s_{2}>s_{1}$ and $f_{\pi}\left(k, i, \sigma(x, k, i), s_{1}+1\right)=1$. Then step (3) holds at stage $s_{2}+1$, and consequently $f\left(z, \sigma(x, k, i), s_{1}+1\right)=0$ for all $z$ other than $2 x$ and $2 x+1$. Beginning from some stage $s_{3}$, the values of the function $\lambda s f_{\pi}(k, i, \sigma(x, k, i), s)$ will stabilize at 1 , and hence $f(z, \sigma(x, k, i), s)=0$ for all $z$ other than $2 x$ and $2 x+1$ and for all $s \geq s_{3}$. Therefore, $\pi_{k}(i)=\alpha(2 x)$ or $\pi_{k}(i)=\alpha(2 x+1)$.

Sufficiency. Let $\langle x\rangle_{0} \neq k$ and $\pi_{k}(i)=\alpha(2 x)$ or $\pi_{k}(i)=\alpha(2 x+1)$. By construction, the number $\sigma(x, k, i)$ is enumerated at step 1 into the sets $\alpha(2 x)$ and $\alpha(2 x+1)$, never to be removed. Consequently, there is a stage $s_{0}$ such that $f_{\pi}(k, i, \sigma(x, k, i), s)=1$ for all $s \geq s_{0}$. Let $s_{1}+1 \in$ $S(x, k, i)$ be the least stage at which $s_{1} \geq s_{0}$. If the value $o(x, k, i)$ has not been defined by stage $s_{1}+1$ then, at this stage, all conditions of step (1) hold, and $o(x, k, i)$ will be set equal to $i$. Lemma 2 is proved.

LEMMA 3. For any $x$ and $z$, if $x \neq z$ then $\alpha(2 x)$ and $\alpha(2 x+1)$ are distinct from all sets $\alpha(z)$ with $z \notin\{2 x, 2 x+1\}$. Moreover, $\alpha(2 x)=\alpha(2 x+1)$ if and only if the procedure $\mathcal{D}(x, i)$ is carried out for all $i$.

Proof. Let $x=\langle k, m\rangle$. If $k \neq 0$, then at step 1 the numbers $\sigma(x, 0, i), i \in \omega$, are enumerated into both sets $\alpha(2 x)$ and $\alpha(2 x+1)$. Since $f_{\pi}(0, i, \sigma(x, 0, i), s)=0$ for all $i$ and $s$, it follows that $o(x, 0, i)$ cannot be defined at any stage of the construction via step (1). Therefore, the numbers $\sigma(x, 0, i)$ cannot be enumerated due to step (2) into any set $\alpha(z)$ for all $z \notin\{2 x, 2 x+1\}$.

If $k=0$ then at step 1 the number $d_{0}(x, 0)$ is enumerated into $\alpha(2 x)$, while $d_{1}(x, 0)$ is enumerated into $\alpha(2 x+1)$. Since $f_{\pi}\left(0, y, d_{0}(x, 0), s\right)=0$ and $f_{\pi}\left(0, y, d_{1}(x, 0), s\right)=0$ for all $y$ and $s$, the values $l(x)$ and $r(x)$ cannot be defined via step (4) at any stage of the construction. Hence steps (5) and (7) cannot be used to enumerate the numbers $d_{0}(x, 0)$ and $d_{1}(x, 0)$ into any set $\alpha(z)$ for all $z \notin\{2 x, 2 x+1\}$.

Thus each of the sets $\alpha(2 x)$ and $\alpha(2 x+1)$ contains a number that is never enumerated into any of the sets $\alpha(z)$ for $z \notin\{2 x, 2 x+1\}$.

To prove the second statement of the lemma, note that

$$
\{\sigma(x, e, i): e, i \in \omega\} \subseteq \alpha(2 x) \cap \alpha(2 x+1),
$$

and that any number of $\alpha(z)$ for $z \notin\{2 x, 2 x+1\}$ is contained in $\alpha(2 x)$ iff it is contained in $\alpha(2 x+1)$. Hence the equality or inequality of the sets $\alpha(2 x)$ and $\alpha(2 x+1)$ is completely determined by the number of stages at which the procedure $\mathcal{D}(x, i)$ is effected. Lemma 3 is proved.

LEMMA 4. If $\pi_{k}$ is a numbering of $\mathcal{A}$, then $\lambda m l(\langle k, m\rangle)$ and $\lambda m r(\langle k, m\rangle)$ are computable functions and

$$
\alpha(2\langle k, m\rangle)=\pi_{k}(l(\langle k, m\rangle)), \alpha(2\langle k, m\rangle+1)=\pi_{k}(r(\langle k, m\rangle)) .
$$


Proof. Let $\pi_{k}$ be a numbering of the family $\mathcal{A}$. We show that the functions $\lambda m l(\langle k, m\rangle)$ and $\lambda m r(\langle k, m\rangle)$ are total. Choose an arbitrary number $m$ and let $x=\langle k, m\rangle$. At step $1, d_{0}(x, 0)$ is enumerated into $\alpha(2 x)$ and $d_{1}(x, 0)$ is enumerated into $\alpha(2 x+1)$, never to be removed. Note that at each stage of the construction, the value $l(x)$ is defined iff the value $r(x)$ is defined.

Assume that both $l(x)$ and $r(x)$ are undefined throughout the construction. Then at each stage $s+1 \in S(x, k, i)$ steps (1)-(9) do not hold, and hence $d_{0}(x, 0)$ is not enumerated into any set $\alpha(z)$ with $z \neq 2 x$, while $d_{1}(x, 0)$ is not enumerated into any set $\alpha(z)$ with $z \neq 2 x+1$. Let $y_{0}$ and $y_{1}$ be $\pi_{k}$-indices of $\alpha(2 x)$ and $\alpha(2 x+1)$. Clearly, $y_{0} \neq y_{1}$. Then $f_{\pi}\left(k, y_{0}, d_{0}(x, 0), s\right)=1$ and $f_{\pi}\left(k, y_{1}, d_{1}(x, 0), s\right)=1$ for all $s$ starting with some $s_{0}$. This implies that the conditions of step (4) hold at infinitely many stages of $S(x, k, i)$, a contradiction. Thus the values $l(x)$ and $r(x)$ are both defined at some stage $s_{1}+1 \in S(x, k, i)$.

We fix a number $i$ and show that $d_{0}(x, i) \notin \alpha(z)$ for all $z \notin\{2 x, 2 x+1\}$. If $d_{0}(x, i)$ is never declared semi-active, then $d_{0}(x, i) \notin \alpha(z)$ for all $z$. If $d_{0}(x, i)$ is declared semi-active at some stage and remains semi-active at all later stages, then $d_{0}(x, i) \in \alpha(2 x)$ and $d_{0}(x, i) \notin \alpha(z)$ for all $z \neq 2 x$.

Consider the case where the number $d_{0}(x, i)$ is declared active at some stage $s_{0}$ and remains active at all stages $s \geq s_{0}$. Then $f\left(2 x, d_{0}(x, i), s\right)=1$ for all $s \geq s_{0}$. If $f\left(2 x+2, d_{0}(x, i), s\right)=0$ for all $s \geq s_{0}$ then $d_{0}(x, i) \notin \alpha(z)$ for all $z$ other than $2 x$ and $2 x+1$.

Let $s_{1}+1$ be the least stage such that $f\left(2 x+2, d_{0}(x, i), s_{1}+1\right)=1$. Then $s_{1}+1>s_{0}$ and step (5) holds at stage $s_{1}+1$; hence $f_{\pi}\left(k, l(x), d_{0}(x, i), s_{1}+1\right)=0$ and $f\left(z, d_{0}(x, i), s_{1}+1\right)=1$ for all $z$. If $f_{\pi}\left(k, l(x), d_{0}(x, i), s+1\right)=0$ for all $s>s_{1}$, then step (6) does not hold at all further stages of $S(x, k, i)$, and $f\left(z, d_{0}(x, i), s+1\right)=1$ for every $z$ and for all $s>s_{1}$. Consequently, the number $d_{0}(x, i)$ is contained in all sets of the family $\mathcal{A}$ but is not contained in $\pi_{k}(l(x))$; therefore, $\pi_{k}$ is not a numbering of $\mathcal{A}$, a contradiction. Hence there is a least stage $s_{2}+1>s_{1}+1$ of $S(x, k, i)$ at which step (6) holds. Then $f\left(z, d_{0}(x, i), s+1\right)=0$ for all $z \neq 2 x$. Thus, at stages $s, s_{1}+1 \leq s \leq s_{2}+1$, the procedure $\mathcal{L}(x, i)$ has been performed completely and the function $\lambda s h_{\pi}\left(k, l(x), d_{0}(x, i), s\right)$ has changed its value at least once. After stage $s_{1}$, therefore, the procedure $\mathcal{L}(x, i)$ can be carried out only finitely many times. Since $\pi_{k}$ is a numbering of $\mathcal{A}$, every execution of this procedure will be finished by fulfilling the instructions of step (6).

If the number $d_{0}(x, i)$ remains active till the end of the construction, then step (9) does not hold at stages in $S(x, k, i)$, and so $\alpha(2 x)$ is the only set in $\mathcal{A}$ that contains $d_{0}(x, i)$. If the number $d_{0}(x, i)$ becomes inactive at some stage $s_{3}+1 \in S(x, k, i)$, then step (9) holds at this stage; i.e., the procedure $\mathcal{D}(x, i)$ is performed. The number $d_{0}(x, i)$ remains inactive at further stages as well. At stages $s>s_{3}+1, d_{0}(x, i)$ can be enumerated into all sets $\alpha(z)$ with $z \notin\{2 x, 2 x+1\}$ via the procedure $\mathcal{L}(x, i)$ only. As shown above, every execution of this procedure will be finished by fulfilling the instructions of step (6). Therefore, the number $d_{0}(x, i)$ is not contained in any set $\alpha(z)$ with $z \notin\{2 x, 2 x+1\}$.

Now we show that $\alpha(2 x)=\pi_{k}(l(x))$. By Lemma $3, \alpha(2 x) \neq \alpha(z)$ for all $z \notin\{2 x, 2 x+1\}$. If, starting with a stage $s^{\prime}$, the number $d_{0}(x, i)$ is active for some $i$, then $d_{0}(x, i) \in \pi_{k}(l(x)) \cap \alpha(2 x)$ 
and $d_{0}(x, i)$ is not contained in any set $\alpha(z)$ with $z \neq 2 x$. Hence $\alpha(2 x)=\pi_{k}(l(x))$.

On the other hand, declaring every number $d_{0}(x, i), i \in \omega$, to be active means that the procedure $\mathcal{D}(x, i)$ will be performed for all $i$. Then $\left\{d_{0}(x, i), d_{1}(x, i): i \in \omega\right\} \subseteq \alpha(2 x) \cap \alpha(2 x+1)$ and no number $d_{0}(x, i)$ is contained in any set $\alpha(z)$ with $z \notin\{2 x, 2 x+1\}$. Furthermore, for every such $z$ and every $y \in \alpha(z)$,

$$
y \in \alpha(2 x) \Longleftrightarrow y \in \alpha(2 x+1) .
$$

Hence $\alpha(2 x)=\alpha(2 x+1)=\pi_{k}(l(x))$.

The equality $\alpha(2 x+1)=\pi_{k}(r(x))$ can be proved similarly. Lemma 4 is completed.

Let

$$
\begin{aligned}
L_{k} & =\left\{l(x):\langle x\rangle_{0}=k\right\}, \\
R_{k} & =\left\{r(x):\langle x\rangle_{0}=k \&\langle l(x), r(x)\rangle \in \varepsilon_{\langle x\rangle_{1}}\right\}, \\
O_{k} & =\left\{o(x, k, i):(x, k, i) \in \operatorname{dom}(o) \&\langle x\rangle_{0} \neq k\right\}, \\
\Pi_{k} & =L_{k} \cup R_{k} \cup O_{k} .
\end{aligned}
$$

LEMMA 5. If $\pi_{k}$ is a numbering of the family $\mathcal{A}$, then $\Pi_{k}$ is a c.e. set that contains exactly one $\pi_{k}$-index of each of the sets $\{\alpha(2\langle k, m\rangle), \alpha(2\langle k, m\rangle+1): m \in \omega\}$ and all $\pi_{k}$-indices of other sets.

Proof. $\Pi_{k}$ is a c.e. set since $l, r$, and $o$ are partial computable functions, and $\varepsilon_{m}$ is a c.e. relation for every $m$. The other statements of the lemma follow from Lemmas 2-4. Lemma 5 is proved.

Now we are in a position to complete the proof of the theorem. By Lemma $1, \alpha$ is a $\Sigma_{a}^{-1}$ computable numbering. Using the criterion given in Theorem 1, we show that the family $\mathcal{A}=\alpha(\omega)$ has no $\Sigma_{a}^{-1}$-computable minimal numbering.

Let $\nu$ be an arbitrary $\Sigma_{a}^{-1}$-computable numbering of $\mathcal{A}$. Then $\nu=\pi_{k}$ for some $k$. Theorem 1 can be reformulated in a more convenient form. Namely:

THEOREM 3. A numbering $\pi_{k}$ of the family $\mathcal{A}$ is not minimal if and only if there is a c.e. set $W$ such that $\pi_{k}(W)=\mathcal{A}$, and for every $m$, one of the following holds:

(a) $\exists u \exists v\left((u, v) \in \varepsilon_{m} \& \pi_{k}(u) \neq \pi_{k}(v)\right)$,

or

(b) $\exists u \forall w \in W\left((u, w) \notin \varepsilon_{m}\right)$.

As the set $W$ we take $\Pi_{k}$. By Lemma $5, \pi_{k}\left(\Pi_{k}\right)=\mathcal{A}$. Let $\varepsilon_{m}$ be an arbitrary c.e. equivalence. Put $x=\langle k, m\rangle$. In view of Lemma 4 , the numbers $l(x)$ and $r(x)$ are defined. There are two cases to consider:

Case 1. Let $\langle r(x), l(x)\rangle \in \varepsilon_{m}$.

Then $\langle r(x), l(x)\rangle \in \varepsilon_{m}^{s+1}$ for all stages $s+1 \in S(x, k, i), i \in \omega$, beginning with some $s_{0}+1$. At these stages, therefore, step (9) of the construction does not hold. Hence the procedure $\mathcal{D}(x, i)$ is performed only for finitely many numbers $i$. By virtue of Lemmas 3 and $4, \pi_{k}(r(x)) \neq \pi(l(x))$. Consequently, Theorem 3(a) holds for $u=r(x)$ and $v=l(x)$. 
Case 2. Let $\langle r(x), l(x)\rangle \notin \varepsilon_{m}$.

We will show that the procedure $\mathcal{D}(x, i)$ is performed for every $i$ in this case. Let $i>0$ be a number such that, at some stage $s_{1}+1 \in S(x, k, i-1)$, the numbers $d_{0}(x, i+1)$ and $d_{1}(x, i+1)$ are declared semi-active. Then the procedure $\mathcal{D}(x, i-1)$ is performed at stage $s_{1}+1$, whereby

$$
f_{\pi}\left(k, l(x), d_{0}(x, i), s_{1}+1\right)=1, f_{\pi}\left(k, r(x), d_{1}(x, i), s_{1}+1\right)=1,
$$

and the numbers $d_{0}(x, i)$ and $d_{1}(x, i)$ are declared active.

We show that the procedure $\mathcal{D}(x, i)$ will be carried out at some later stage in $S(x, k, i)$. Lemma 4 shows that if step (5) (step (7)) holds at some stage in $S(x, k, i)$, then step (6) (step (8)) will hold at some later stage in $S(x, k, i)$. Therefore, for some $s_{2}+1 \in S(x, k, i)$,

$$
\begin{gathered}
f_{\pi}\left(k, l(x), d_{0}(x, i), s+1\right)=1, f_{\pi}\left(k, r(x), d_{1}(x, i), s+1\right)=1, \\
f\left(2 x, d_{1}(x, i), s\right)=0, f\left(2 x+1, d_{0}(x, i), s\right)=0
\end{gathered}
$$

for all $s+1 \in S(x, k, i)$ with $s \geq s_{2}$. By Lemma $4, \alpha(2 x)=\pi_{k}(l(x)), \alpha(2 x+1)=\pi_{k}(r(x))$, and, as long as the numbers $d_{0}(x, i+1)$ and $d_{1}(x, i+1)$ remain semi-active, they cannot be enumerated into sets distinct from $\alpha(2 x)$ and $\alpha(2 x+1)$, respectively. Therefore, for some $s_{3} \geq s_{2}$,

$$
f_{\pi}\left(k, l(x), d_{0}(x, i+1), s+1\right)=1, f_{\pi}\left(k, r(x), d_{1}(x, i+1), s+1\right)=1
$$

for all $s+1 \in S(x, k, i)$ after stage $s_{3}$, as long as $d_{0}(x, i+1)$ and $d_{1}(x, i+1)$ remain semi-active. Hence step (9) is effected at a least stage $s_{4}+1 \geq s_{3}$ in $S(x, k, i)$. Then, at this stage, the procedure $\mathcal{D}(x, i)$ is carried out.

Thus $\langle r(x), l(x)\rangle \notin \varepsilon_{m}$ and the procedure $\mathcal{D}(x, i)$ will be executed for every $i$. Then $r(x) \notin \Pi_{k}$, and by Lemmas 3 and $4, \pi_{k}(r(x))=\pi(l(x))$. If $\langle r(x), w\rangle \notin \varepsilon_{m}$ for all $w \in \Pi_{k}$ distinct from $l(x)$, then condition (b) of Theorem 3 holds for $u=r(x)$ and for all $w \in \Pi_{k}$. If, however, $\langle u, w\rangle \notin \varepsilon_{m}$ for some $w \in \Pi_{k}$ distinct from $l(x)$, then, by Lemma 5 , condition (a) of Theorem 3 holds for $u=r(x)$ and $v=w$.

Thus at least one of the conditions (a) or (b) specified in Theorem 3 holds in either case. Consequently, $\pi_{k}$ is not a minimal numbering. Theorem 2 is proved.

\section{REFERENCES}

1. V. V. V'yugin, "On some examples of upper semilattices of computable enumerations," Algebra and Logic, 12, No. 5, 277-286 (1973).

2. S. A. Badaev, "Minimal enumerations," Sib. Adv. Math., 2, No. 1, 1-30 (1992).

3. S. A. Badaev and Zh. T. Talasbaeva, "Computable numberings in the hierarchy of Ershov," Mathematical Logic in Asia, S. S. Goncharov (Ed.), World Scientific, NJ, 17-30 (2006).

4. S. A. Badaev and S. Lempp, "A decomposition of the Rogers semilattice of a family of d.c.e. sets," J. Symb. Log., 74, No. 2, 618-640 (2009). 
5. A. B. Khutoretskii, "On the cardinality of the upper semilattice of computable enumerations," Algebra and Logic, 10, No. 5, 348-352 (1971).

6. K. Sh. Abeshev, "On the existence of universal numberings for finite families of d.c.e. sets," Math. Log. Q., 60, No. 3, 161-167 (2014).

7. S. A. Badaev and S. S. Goncharov, "Theory of numberings: Open problems," in Computability Theory and Its Applications, Current Trends and Open Problems, Cont. Math., 257, S. Cholak et al. (Eds.), Am. Math. Soc., Providence, RI (2000), pp. 23-38.

8. H. Rogers, Theory of Recursive Functions and Effective Computability, McGraw-Hill, New York (1967).

9. Yu. L. Ershov, "A hierarchy of sets. I," Algebra and Logic, 7, No. 1, 25-43 (1968).

10. Yu. L. Ershov, "On a hierarchy of sets, II," Algebra and Logic, 7, No. 4, 212-232 (1968).

11. Yu. L. Ershov, "On a hierarchy of sets. III," Algebra and Logic, 9, No. 1, 20-31 (1970).

12. C. J. Ash and J. F. Knight, Computable Structures and the Hyperarithmetical Hierarchy, Stud. Log. Found. Math., 144, Elsevier, Amsterdam (2000).

13. S. S. Goncharov and A. Sorbi, "Generalized computable numerations and nontrivial Rogers semilattices," Algebra and Logic, 36, No. 6, 359-369 (1997).

14. Yu. L. Ershov, The Theory of Numberings [in Russian], Nauka, Moscow (1977). 